\title{
Fault identification of double-circuit transmission lines on the same pole based on EEMD energy ratio
}

\author{
Yicen Liu ${ }^{1}$, Ying Chen ${ }^{2}$, Songhai Fan ${ }^{1}$, Yiyu Gong ${ }^{1}$, and Xi Zou ${ }^{3, *}$ \\ ${ }^{1}$ State Grid Sichuan Electric Power Research Institute, Chengdu, Sichuan, 610041, China \\ ${ }^{2}$ State Grid Sichuan Integrated Energy Service Co., Ltd., Chengdu, Sichuan, 610072, China \\ ${ }^{3}$ Automation and Information Engineering, Sichuan University of Science \& Engineering, zigong, \\ Sichuan, 643000, China
}

\begin{abstract}
In order to improve the sensitivity and reliability of traveling wave protection, on the basis of analyzing the relationship of the antitraveling wave current amplitude in the window after the internal/external failure of the double circuit line on the same tower, a fault identification method based on EEMD energy ratio is proposed. Use EEMD decomposition to decompose the anti-traveling wave current in a time window after the fault into 7 scales, and extracts the EEMD energy ratio at each scale at both ends to form a feature vector. Then it is sent to the particle swarm optimization support vector machine (PSO-SVM) for training and testing, and the internal and external faults are identified. Experiments show that the algorithm has good fault identification ability, the fault accuracy is $95 \%$ and the method sensitivity is high.
\end{abstract}

Keywords: Double-circuit lines on the same tower, Reverse traveling wave current, EEMD energy ratio, PSO-SVM, Fault identification.

\section{Introduction}

At present, double-circuit transmission lines on the same pole are used more and more widely in high-voltage transmission lines. This makes its fault conditions become very complicated, and the damage after the fault occurs will be more serious, so it is necessary to solve the faulty circuit in time to avoid major economic losses.

Many scholars have done a lot of research on the same pole double circuit transmission line [1]. Jiang Xianguo et al. [2] proposed an anti-misoperation scheme for zero-sequence pilot protection. First, by analyzing the misoperation mechanism of the zero-sequence pilot protection of a normal line during the one-circuit disconnection of the transmission line, the line fault identification criterion was proposed. Use the phase and amplitude of the load current before the fault and the zero sequence current after the fault to distinguish the two types of faults. Wang Ze yang et al. [3] further studied the cross-differential protection of the double-circuit line on the same pole, and proposed a six-sequence cross-differential

*Corresponding author: zouxi647636@163.com 
protection method for the traditional cross-differential protection that uses the non-fault phase-to-phase voltage as the reference vector.

\section{Analysis of fault traveling wave transmission characteristics}

Figure 1 shows the double circuit line model on the same pole. $\mathrm{M}$ and $\mathrm{N}$ are the bus bars at both ends; L1 and L2 are two-circuit lines; PM and NO are used to indicate the two outer lines of L3 and L4; R1-R6 are close to each other. The protective element at the busbar end. $\mathrm{K} 1$ and $\mathrm{K} 2$ are the locations of the fault points, which are the internal fault and the external fault respectively, and the opposite direction is the bus outflow line.

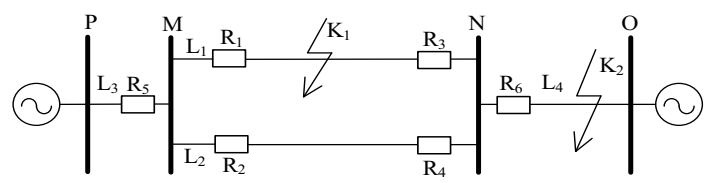

Fig. 1. Double circuit line model on the same pole.

\subsection{Internal and external failure analysis}

Through the superposition theorem, the generated fault can be regarded as the superposition of the normal state and the additional state of the fault for discussion [4]. The fault component state can be regarded as adding a power source at the fault point, and a fault traveling wave will be generated at the fault point for transmission to both ends of the line.

Figure 2 and Figure 3 show the propagation process of traveling waves when faults occur outside and inside the area. Taking a single-phase fault as an example, Figure 2 shows the propagation process of traveling waves when a fault occurs outside the zone, and Figure 3 shows the propagation process of a fault within the zone.

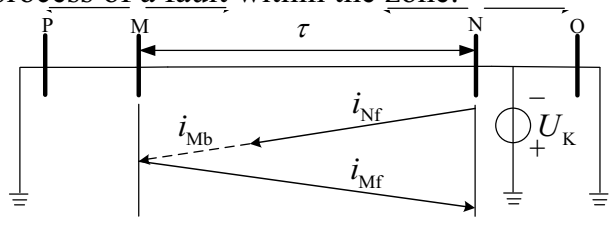

Fig. 2. Travelling wave propagation process during faults outside the zone.

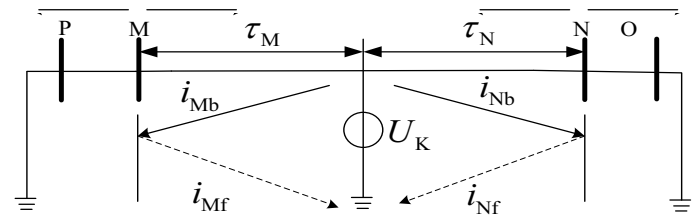

Fig. 3. Traveling wave propagation process in the event of a fault in the zone.

The calculation formula of the corresponding traveling wave current is shown in the following formula (1):

$$
\left\{\begin{array}{l}
i_{M b}=-i_{M}+\frac{u_{M}}{Z_{\mathrm{c}}} \\
i_{N b}=-i_{N}+\frac{u_{N}}{Z_{\mathrm{c}}}
\end{array}\right.
$$

where: $Z_{c}$ is the line wave impedance, $u_{M}, u_{N}, i_{M}, i_{N}$ are the voltage and current at both ends. 


\section{Algorithm implementation}

The traveling wave current and voltage at both ends of the line are decoupled by phase-mode transformation, the same modulus is selected to calculate the current of the anti-traveling wave modulus at both ends, the modulus current is decomposed into 7 scale frequency bands using EEMD, and each scale is calculated After downloading the EEMD energy value in $[\tau, 2 \tau)$ after the fault, the EEMD energy ratio feature vectors at each scale at both ends are then used to form the training sample, and then the PSO-SVM is used for training and classification to identify the fault.

\subsection{Phase-to-mode conversion}

In the double-circuit transmission line on the same pole, there is the influence of line coupling, so the line needs to be decoupled, and the phase-to-mode transformation matrix is usually used for decoupling, turning it into a mutually independent single-phase system. This article uses the phase-to-mode transformation method proposed by Wang Shoupeng et al. [5].

\subsection{EEMD decomposition}

EEMD can decompose almost all signals into several IMF components according to the characteristics of the signal itself, which is a very effective method for processing nonlinear signals. Equation (2) is the newly formed signal. After EMD decomposition, the newly formed signal can be expressed by the sum of the IMF component and the remainder [6].

$$
x_{i}(t)=x(t)+n_{i}(t)
$$

In the formula, $n_{i}(t)$ is the white noise added for the $i$ time; $x(t)$ the original input signal; $x_{i}(t)$ is the new signal formed by adding white noise for the $i$ time, $i=1,2 \cdots, N$.

$$
x(t)=\sum_{i=1}^{n} R_{i}+c_{n}(t)
$$

In the formula, $R_{i}$ is the IMF component of each order, $i$ represents the order, $c_{n}(t)$ is the residual term, and $n$ is the number of $R_{i}$.

\subsection{Analysis of anti-traveling wave current in internal and external faults}

\subsubsection{Analysis of backward travelling wave current in faults of single circuit lines in the district}

The reverse traveling wave current at both ends of the L1 line A and B phases in the protection zone after a short-circuit grounding fault occurs within $[\tau, 2 \tau)$, as shown in Figure 4 , Figure 5 and Figure 6 . Figure 4 shows the anti-traveling wave currents at both ends, and Figures 5 and 6 show the frequency scales corresponding to the EEMD decomposition of the $\mathrm{M}$ and $\mathrm{N}$ ends. 


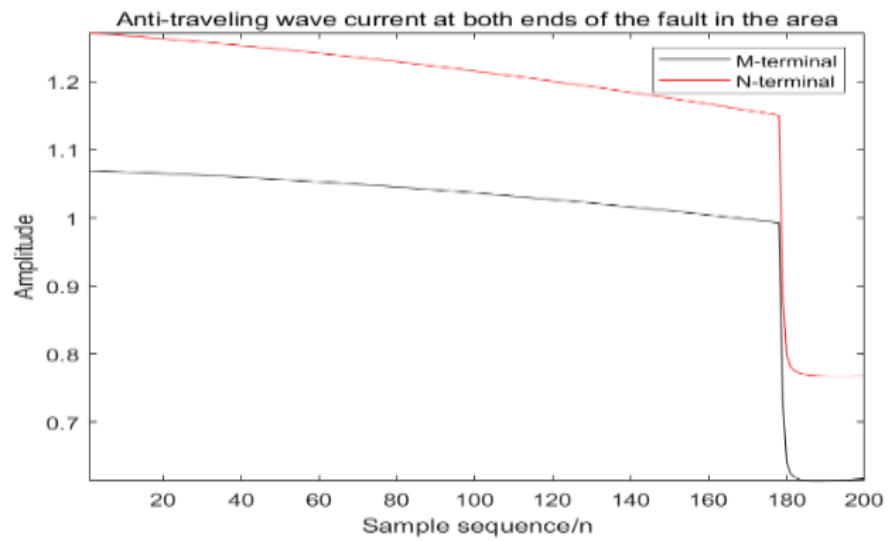

Fig. 4 Current waveforms at both ends of a single-circuit fault in the area.

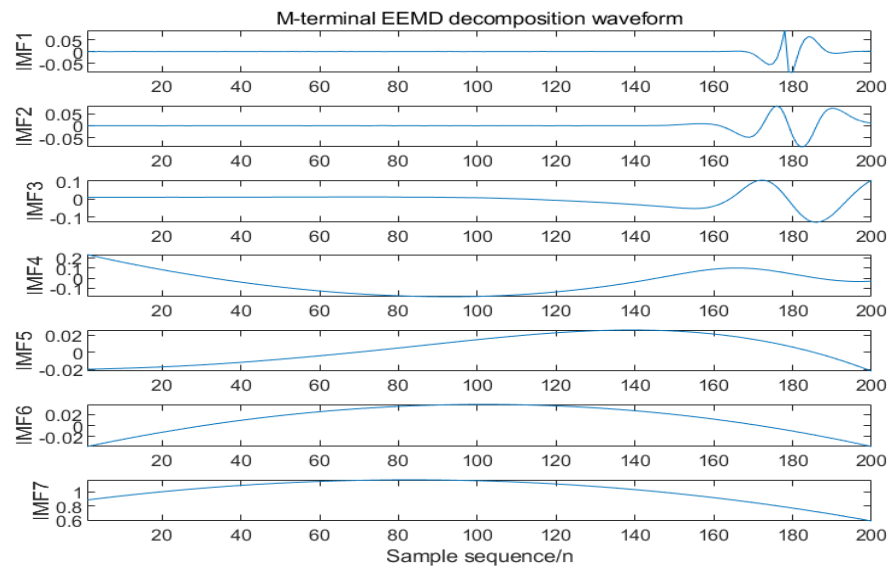

Fig. 5. Decomposition of EEMD at $M$ terminal of single-circuit fault in the area.

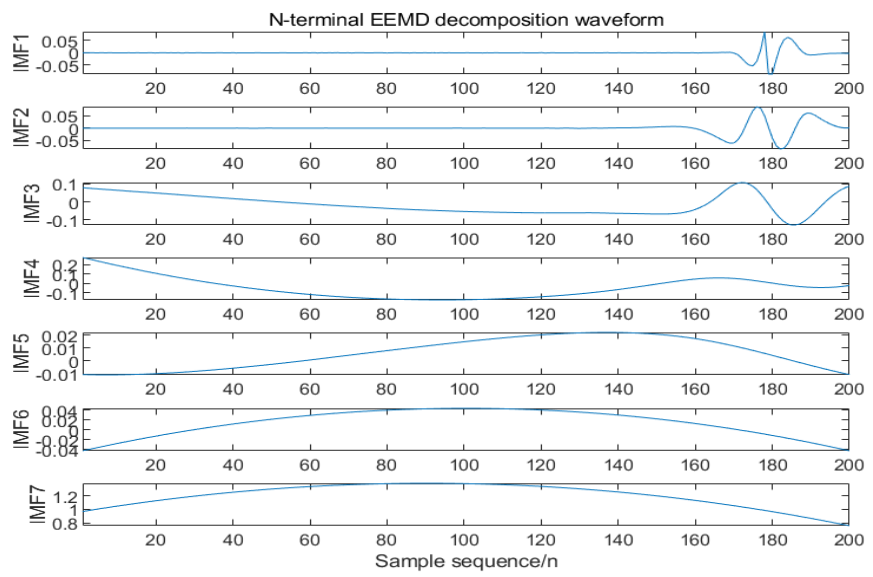

Fig. 6. N-side EEMD decomposition of a single-circuit fault in the area.

Figure 4 shows that in the time after the fault $[\tau, 2 \tau)$, both ends generate anti-traveling wave signals. After the anti-traveling wave current is decomposed by EEMD, it can be seen 
from Figures 5 and 6 , that $\mathrm{M}$ The currents of the terminal and the $\mathrm{N}$ terminal are relatively close, and the energy difference is not very large.

\subsubsection{Analysis of anti-traveling wave current in out-of-area faults}

The waveforms of the anti-traveling wave currents at both ends within $[\tau, 2 \tau)$ after a shortcircuit grounding fault occurs on phases A and B outside the area are shown in Figure 7. Figures 8 and 9 show the frequency scales corresponding to the EEMD decomposition of the $\mathrm{M}$ and $\mathrm{N}$ ends.

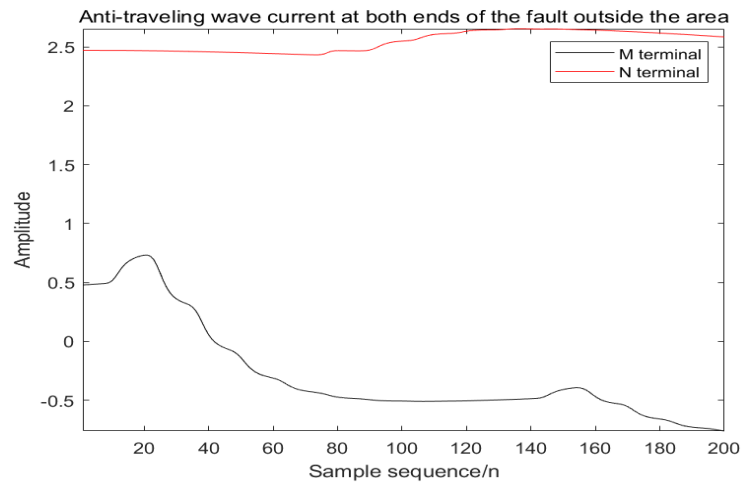

Fig. 7. Anti-traveling wave current at both ends of the fault outside the zone.

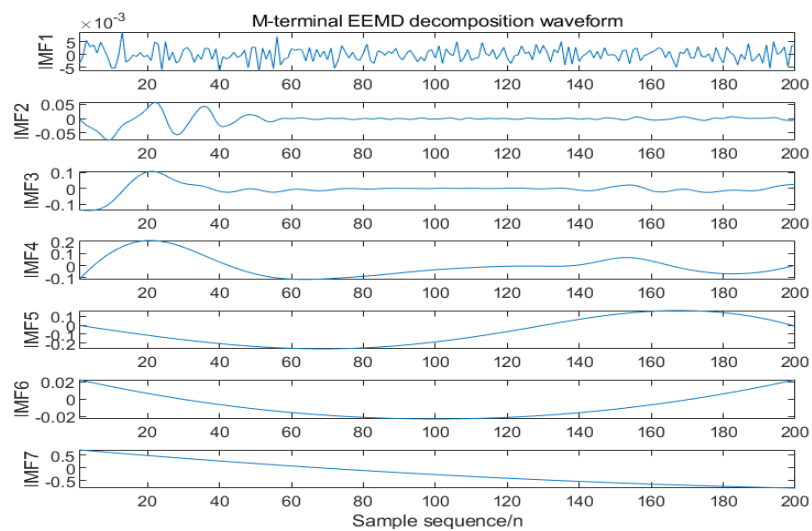

Fig. 8. EEMD decomposition waveform of M-side fault outside the zone.

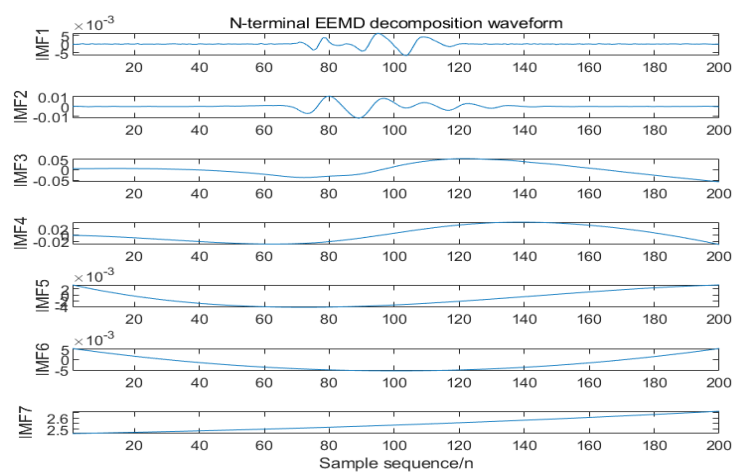

Fig. 9. Decomposed waveform of N-terminal EEMD of out-of-zone fault. 
Figure 7 shows that in the time after the fault $[\tau, 2 \tau)$, both ends generate anti-traveling wave signals. After the anti-traveling wave current is decomposed by EEMD, it can be seen from Figures 8 and 9, M The current magnitude difference between the terminal and the $\mathrm{N}$ terminal is relatively large, and the energy difference is also relatively large.

\subsection{EEMD energy ratio fault feature extraction}

After EEMD decomposition, the fault signals distributed in different frequency bands can be obtained. The signal energy of these frequency bands contains a lot of fault information, which can be used for fault identification.

By integrating the coefficient square of each frequency band, the integral value is taken as the energy value of the scale. The expression is shown in the following formula (4):

$$
\left\{\begin{array}{l}
E_{M n}=\int_{t=t_{0}+\tau}^{t_{0}+2 \tau}\left|W_{M n}(t)\right|^{2} \\
E_{N n}=\int_{t=t_{0}+\tau}^{t_{0}+2 \tau}\left|W_{N n}(t)\right|^{2}
\end{array}\right.
$$

where: $E_{M n}, E_{N n}$ are the EEMD energy of the nth layer at the $\mathrm{M}$ and $\mathrm{N}$ terminals respectively; $W_{M n}(t), W_{N n}(t)$ are the EEMD transformation coefficients of the nth layer at the $\mathrm{M}$ and $\mathrm{N}$ terminals; $t_{0}$ is the time when the fault occurs; $\tau$ is the traveling wave transmitted from one end of the bus to the other end Time; $\tau$ is $1 \mathrm{~ms}$.

The EEMD energy ratio calculation formula is:

$$
P_{n}=\frac{\max \left(E_{M n}, E_{N n}\right)}{\min \left(E_{M n}, E_{N n}\right)}
$$

$P_{n}$ is the anti-traveling wave current energy ratio of the nth layer. The sequence is:

$$
P=\left[P_{1}, P_{2}, \cdots, P_{n}, \cdots\right]
$$

Decomposing the anti-traveling wave EEMD to get the energy of 7 scales to calculate the energy ratio and input it as the feature vector $\left[P_{1}, P_{2}, P_{3}, P_{4}, P_{5}, P_{6}, P_{7}\right]$ into the support vector machine for fault identification.

\section{Fault identification algorithm based on PSO-SVM}

\subsection{Support vector machines}

Support vector machine (SVM) is mainly used to solve the data classification problem in the field of pattern recognition, which belongs to a kind of supervised learning algorithm. It can transform nonlinear problems into linear problems, which greatly improves our computational efficiency.

\subsection{Particle optimization swarm algorithm}

The PSO algorithm is an evolutionary computing technology, and its basic idea is to find the optimal solution through collaboration and information sharing between individuals in the group.

The advantage of PSO is that it is simple and easy to implement and does not have many parameter adjustments. It has been widely used in function optimization, neural network training, fuzzy system control and other genetic algorithm applications. 


\subsection{Algorithm flow}

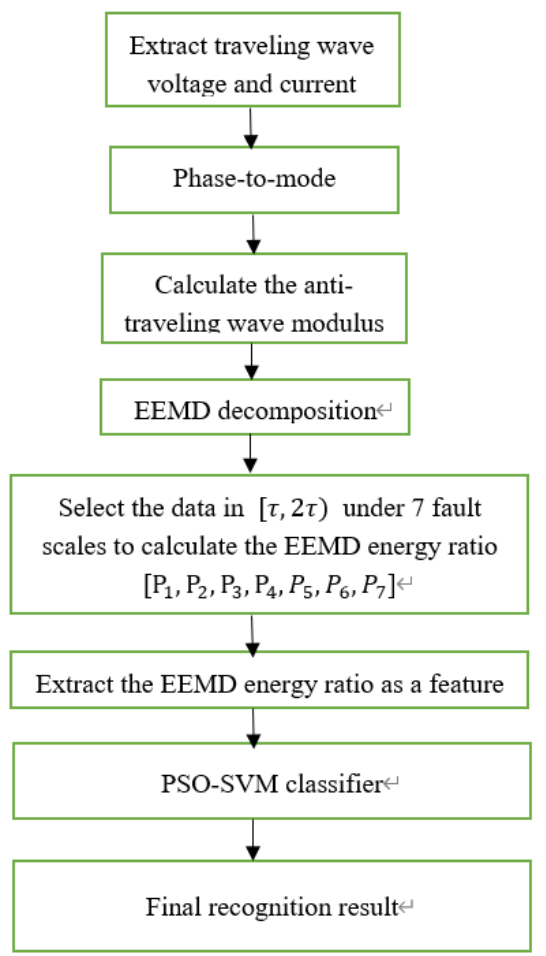

Fig. 10. Fault identification process.

When the transmission line fails, the current and voltage are converted into phase mode; the same modulus is selected to calculate the traveling wave current at both ends; the reverse traveling wave modulus within $[\tau, 2 \tau)$ time after the fault is selected for EEMD decomposition, Then calculate the EEMD energy at 7 scales, and then calculate the EEMD energy ratio; extract 7 EEMD energy ratios $\left[\mathrm{P}_{1}, \mathrm{P}_{2}, \mathrm{P}_{3}, \mathrm{P}_{4}, P_{5}, P_{6}, P_{7}\right]$ as feature vectors, and train them to PSO-SVM Carry out classification to realize fault identification. Figure 10 shows the flow chart of fault identification.

\section{Simulation}

PSCAD software is used to establish a double-circuit line model on the same pole. The model is shown in Figure 1. The line MN in the protection zone is $300 \mathrm{~km}$ long, and the line NO outside the protection zone is $150 \mathrm{~km}$ long. The system sampling is $200 \mathrm{kHz}$; the voltage level is $500 \mathrm{kV}$, and the frequency is $50 \mathrm{~Hz}$.

\subsection{Training set composition}

The support vector machine training set is composed of the internal fault samples on the MN line and the external fault samples on the NO line.

(1) In-area fault samples: It is composed of 1170 sets of data obtained by simulating 117 different fault types and 10 different initial fault angles at $150 \mathrm{~km}$ from the $\mathrm{N}$-terminal bus on the MN line. 
(2) Out-of-zone fault samples: 110 sets of data obtained by simulation under 11 different types of faults, 10 different initial angles of faults, and $100 \mathrm{~km}$ away from the N-terminal bus on the NO line.

\subsection{Prediction results of training set and test set}

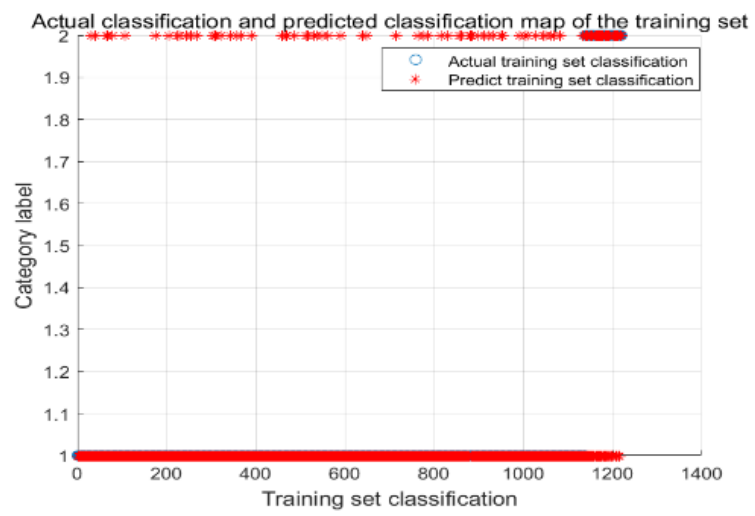

Fig. 11. Training set samples.

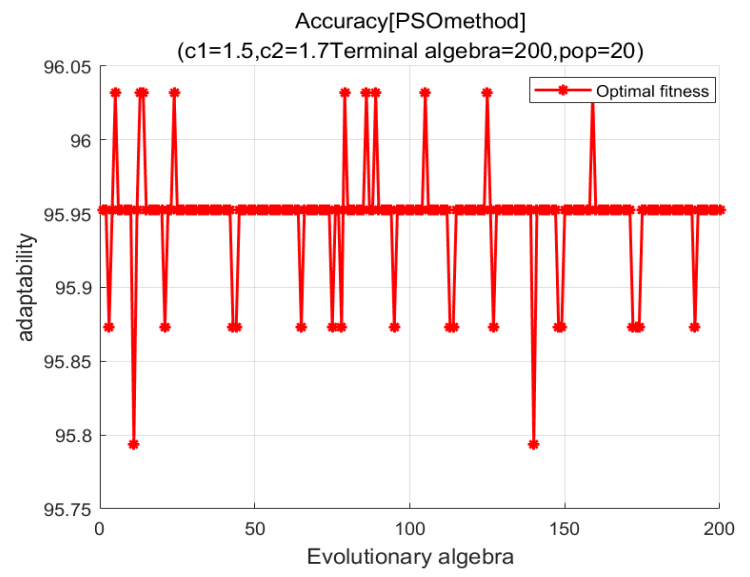

Fig. 12 PSO algorithm fitness curve.

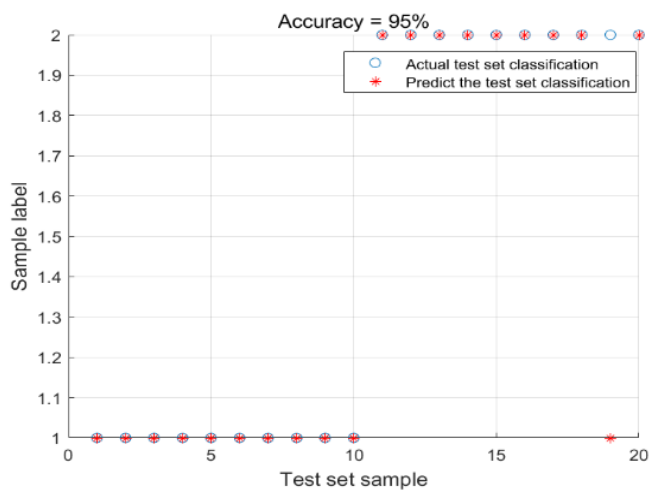

Fig. 13 Test set sample. 
Figure 11 is the recognition result of the training sample by the PSO-SVM model. Label 1 is the fault in the area, and label 2 is the fault outside the area. Figure 12 is the adaptation curve of the PSO algorithm. The learning factor is $c_{1}=1.5, c_{2}=1.7$; the termination algebra is 200 ; Best $\mathrm{c}=78.146$ Best $\mathrm{g}=1000$. Figure 13 shows the test samples. 10 faults in the area and 10 faults outside the area are selected as the test set samples, and the fault classification accuracy rate is $95 \%$.

\section{Summarize}

The following conclusions are obtained through simulation verification. When a fault occurs in the area, the protection unit can detect the anti-traveling wave within $[\tau, 2 \tau)$ after the fault. It is also small; when an out-of-area fault occurs, the protection unit can also detect the antitraveling wave after the failure, but the difference in amplitude of the anti-traveling wave at both ends is relatively large, and the energy ratio is also large.

Project Supported by Science and Technology Project of State Grid Sichuan Electric Power Company (Contract No.521997180016).

\section{References}

1. Zhu Yongli, Fan Guochen, Zhao Xuesong, Chen Hua, Xiong Xi. Four-point traveling wave fault location method for double-circuit transmission lines on the same pole[J]. Journal of Electric Power System and Automation, 2016, 28(12): 124 -129.

2. Jiang Xianguo,Zhou Zexin,Du Dingxiang,Li Zhongqing.An anti-misoperation scheme for zero-sequence longitudinal protection of double-circuit lines on the same tower based on disconnection recognition [J]. Journal of Electric Power System and Automation, 2018,30(11):32-38.

3. Wang Zeyang. Cross-differential protection for double-circuit lines on the same tower based on six-sequence fault components[J].Communication Power Technology,2019,36(12):12-14.

4. Dai Zhihui, Zhang Cheng, Liu Ningning, et al. Pilot protection scheme for UHV DC lines based on the difference of antitraveling waves[J]. Power System Protection and Control, 2019, 47(21):1-10.

5. Wang Shoupeng, Zhao Dongmei, Yuan Jingzhong, et al. A new phase-to-mode transformation method for fault location of double-circuit lines on the same tower [J]. Journal of Xi'an University of Technology

6. Ma Hongwei, Zhang Dawei, Cao Xiangang, et al. Research on vibration signal denoising method based on EMD [J] .Vibration and Shock, 2016, 35(22): 38-40 